\title{
Personalized medicine: current status and prospects
}

\author{
Nasadyuk C. M., Sklyarov O. Ya. \\ Danylo Halytsky Lviv National Medical University, Lviv, Ukraine \\ e-mail: nasadyukch@gmail.com
}

\section{ABSTRACT}

\begin{abstract}
The article presents modern view at the personalized (precision) medicine and discusses the role of genome, glycobiome, microbiome studies in the selection of individualized treatment. Actual data on the options of target immunotherapy of cancer are presented. Up-to-date feasibility of personalized therapy and perspectives for its development are considered.
\end{abstract}

KEY WORDS: personalized medicine; precision medicine; genome; glycobiome; microbiome

The development of genetics, molecular and cell biology changed the ideas about the nature of many diseases, approaches to their treatment and prophylaxis. Despite the introduction of standardized international protocols of different diseases treatment, a novel direction has been actively developing in the up-to-date science - personalized or precision medicine $[3,9,10]$. The latter implicates the individual approach to the diseases treatment, based on the results of investigations of genome, proteome, glycobiome, microbiome of each individual patient. $[3,4,5]$.

Actually, personalized medicine is mainly used in small groups of patients with rare diseases or in the case of ineffectiveness of conventional methods of treatment or contraindications to them $[4,10]$. The main vectors for personalized medicine are cancer, obesity, bronchial asthma, diabetes mellitus, psychic disorders, gout etc. [7, 15].

The emergence of personalized medicine is related with the insufficient effectiveness of the conventional methods of treatment, since it is known, in particular, that the efficacy of antidepressants application makes not more than $40 \%$, antitumor therapy - $75 \%$ and efforts of the scientists to predict the effect of pharmaceutical drugs in certain patients $[15,24]$. To the opinion of scientists, the creation of the large databases according to the results of large population genome investigations will allow to model the transcriptomic profile of drugs [24].

Personalized medicine is a logical result of the actual today tendency to reductionism in medical practice, when the diseases is increasingly often analyzed on the molecular level [5]. The results of molecular investigations and not the origin of tumor and stage of the disease have already become determinating at the selection of chemotherapy in oncologic patients [25].

The development of the personalized medicine is also facilitated by the increase of the number of biobanks worldwide, storing the umbilical cord blood stem cells, other human tissues and cells, which may be used with the purpose of autologous therapy. Personal storage of the biological material is considered to be a kind of bioinsurance and becomes increasingly popular in the countries with high social standards.

\section{HISTORICAL NOTE}

In the scientific publications, the term "personalized medicine" for the first time appeared in 1999, although the conception of personalized medicine was formulated yet in the early 1960-s [10]. Till now the disputes have been lasting on which term is the most correct "personalized medicine" or "precision medicine" and despite the fact that these definitions are interchangeable, the statement of meaning "precision medicine" is used predominantly in scientific sense, whereas "personalized medicine" rather in clinical practice [9].

In 2016, the term "personalized medicine" was officially approved by the National Health Service of England and "precision medicine" in 2015 [9]. Hence, the United Kingdom of Great Britain and Northern Ireland became the first state to introduce personalized medicine into the practical health care [9]. Today, personalized medicine has been actively developing, what is facilitated by the commercialization of genomics, proteomics, bioinformatics, biobanking.

\section{ROLE OF GENOME IN PERSONALIZED MEDICINE}

The main principle of the personalized medicine is the selection of the individual scheme of treatment, based on the determination of the correlation between the genotype of the individual patient and data on the phenotype of their disease [3]. This became possible courtesy of the creation of large electronic databases, containing the results of genotyping of huge cohorts of patients [3]. Up-to-date, more than 6,000 rare diseases were described, $80 \%$ of which are genetically mediated [6]. The best example of the personalized medicine is a use of the drug, potentiating the transmembrane conductivity, to which only $5 \%$ of cystic fibrosis patients are sensitive, having the mutation of gene p.Gly551Asp [4]. Other examples of the successful personalized therapy may include low carbohydrate diet, allowing to achieve clinical improvement at the deficiency of the protein glucose transporter type 1 (syndrome of Glut-1 deficiency) [6]. The established mutation of gene CTPS1 (cytidine triphosphate synthase-1) explains the tendency to severe form of the chickenpox 
infection and allows to administer such patients a bone marrow transplant as a potential therapy [6].

In recent decade, a significant advancement has been made in the understanding of the genetic background of the gout and pharmacogenetics of the hypouricemic therapy. The cohort studies, that enrolled 110,000 Europeans allowed to reveal 28 loci, responsible for the hyperuricemia and it was shown that the efficacy of treatment with alopurinol is mediated by the ABCG2 genes variants [7]. These findings could have practical significance in the treatment of gout if the cost of genetic investigations was lower $[4,7]$.

Genetic variants affect the pharmacokinetics of the drugs, that is important at dosing of pharmaceuticals. Mutations of protein genes, responsible for the transport of statins decrease the effectiveness of hypocholesterolemia therapy and in patients with CYP2D6 gene defect the decreased sensitivity to the drug tamoxifen is noted, the latter is used in the treatment of estrogen-receptor positive breast carcinomas [15].

The determination of the major histocompatibility system (MHC) loci has been increasingly used in the up-to-date personalized medicine, allowing to determinate the inherited tendency to certain diseases, to predict the effectiveness of different methods of treatment and their side effects [13].

However, the personalized medicine has gained the widest use in the preventive oncology, where screening programs are based on the determination of gene mutations. It was established, namely, that the incidence of breast cancer development in female carriers of BRCA1 or BRCA2 gene mutation makes $45-65 \%$ till the age of 70 , and in the case of mutation of APC (adenomatous polyposis coli) gene colon cancer develops till 40-50 years of age of the carrier [20]. In both cases organ resection is considered for the malignancy prevention. Genome associations with rheumatoid arthritis are also well elucidated today [13]

\section{THE ROLE OF GLYCOBIOME IN PERSONALIZED MEDICINE}

Besides genetic research, in recent years glycome studies have been supposed to be extremely promising. The glycome means the set of glycoconjugates, expressed by cells and tissues [14]. Every cell is covered by a carbohydrate layer - glycocalyx, rich and diverse according to its content, consisting mainly of glycoproteins, glycolipids and proteoglycans, involved in numerous reactions of intercellular interaction and response to infectious agents as well as processes of differentiation, apoptosis etc. $[1,2,11]$. The variability of glycoconjugates was proved to be significantly higher than the human genome and as of today less than $2 \%$ of genes were revealed, coding proteins, engaged in glycosylation processes [1].

The acute need for the elucidation of the glycosylation markers, changed by the disease, was substantiated by the scientists with the purpose of more individualized and optimized therapy on the early stages of numerous diseases [1, 11]. Evaluation of protein glycosylation changes also may be useful for the monitoring of the course of disease and efficacy of treatment, in particular in disorders, associated with aging processes [2]. The importance of glycosylation processes research in health and pathology is confirmed by the fact that all FDA approved tumor markers, in particular cancer-embryonic antigen (CEA), alfa-fetoprotein (AFP), mucin 16 (MUC-16), prostate-specific antigen (PSA), $\beta$-chorionic ( $\beta \mathrm{hCG}$ ) and others, are glycoproteins according to their chemical structure [1]. This is because the transformation of a healthy cell into the malignant one is accompanied by the excessive expression of erroneously glycosylated proteins, loss of cell polarity by the epitheliocytes and secretion of proteins with changed glycosylation into the blood flow [1].

More than 50 diseases, mediated by aberrant glycosylation have been discovered within recent 20 years, mediated by the disorders of cell membrane glycoconjugates metabolism, including carbohydrate-deficient glycoprotein syndrome and one of the forms of alcoholism [11]. Glycosilation changes, in particular enhancement of fucosylation and syalation of serum glycoproteins, were noted in the cancer of stomach, colon, ovaries, breasts, prostate, lungs [1].

\section{THE ROLE OF MICROBIOME IN PERSONALIZED MIEDICINE}

According to the results of deeper studies on the role of human microflora in homeostasis and pathogenesis of many diseases (obesity, metabolic syndrome, Crohn's disease, ulcerative colitis, atherosclerosis etc.) bacteria may be considered to be an individual organ and vector of influence by therapeutic measures [8]. Human microbiome is called the second genome, since it encompasses trillions of bacteria, hosting the organism and being integrated with it [16].

The mechanism of the pathogenetic influence of dysbiosis on the development of disease is poorly studied and main theories are based on the change of digestive activity of microbiota and alteration of the intestinal signal pathways, caused by the disbalance of the intestinal metabolites, signaling chemical compounds of low molecular weight, secreted by the bacteria into the intestinal lumen, in particular secondary bile acids, short-chain fatty acids [21].

The role of microbiome in recent years has been widely studied in the interrelationship with obesity and modulation of the patient's intestinal microflora has been increasingly considered as a method of body weight correction [23]. The decrease of the body weight index in obesity patients was shown under the conditions of certain probiotics administration [23].

Up-to-date, the intestinal microflora is known to be involved in the realization of the effectiveness and toxicity of drugs, in particular it was established that intestinal microflora courtesy of its high metabolic activity may inhibit the effect of cytostatic and cardiac glycoside digoxin, and trimethylamine oxide, the product of bacterial metabolism of choline and carnitine, participates in the pathogenesis of atherosclerosis and may be detected as a marker of cardiovascular diseases [8].

One of the directions of the modern personalized medicine is fecal microbiota transplantation in the treatment of inflammatory bowel disease, metabolic syndrome, infectious diseases of the intestine [21]. Fecal microbiota transplantation is approved as a safe and effective method of treatment of recurrent pseudomembranous colitis, caused by Clostridium difficile [22]. Efficacy of fecal microbiota transplantation in the treatment of the metabolic syndrome was shown in experimental studies [21]. However no convincing data on the efficacy of fecal microbiota transplantation in the treatment of Crohn's disease and ulcerative colitis has been obtained [21].

\section{TARGET IMIMUNOTHERAPY OF CANCER}

The personalized methods of treatment have gained the widest application in oncology and mainly are based on the application of the inhibitors of tyrosine kinases and other monoclonal antibodies as well as adoptive immunotherapy with T-lymphocytes [15]. The discoveries in the field of target immunotherapy of cancer with the use of T-lymphocytes were awarded the Nobel Prize in Medicine and Physiology in 2018.

The application of T-lymphocytes with the chimeric antigen receptor, so called CAR-T-lymphocytes is considered to be the most promising method of personalized therapy of oncologic diseases [17]. CAR-T-lymphocytes are obtained with the method of gene editing according to the technology of clustered regularly interspaced short palindromic repeats (CRISPR) from the own T-lymphocytes of the patient [18]. CAR-T-lymphocytes, retransfused back to the patient recognize the superficial antigens of the tumor independently from the major histocompatibility system, proliferate and destroy tumor cells according to the principle of the reaction with the antigen [17]. More than 500 clinical trials, evaluating the safety and efficacy of CAR-T-lymphocytes have been registered in the international database [19]. B-cell lymphomas are supposed to be the most promising indication to CAR-T-cell therapy, that is mediated by the selective and homogenous expression of CD19 or CD20 antigens by them and easier access for T-lymphocytes [17].

\section{CONCLUSIONS AND PROSPECTS FOR THE DEVELOPMENT \\ OF PERSONALIZED MEDICINE}

Results of up-to-date studies show the penetration of personalized treatment methods into the different fields of medicine, being the therapy 
of choice for patients with the established molecular defect, being the background for the disease pathogenesis. The development of personalized medicine will be facilitated by the extending of the electronic da- tabases of genome, glycobiome, microbiome in different disorders and their international integration. The disadvantage of the personalized medicine is its high cost.

\section{REFERENCES}

1. Almeida A, Kolarich D. The promise of protein glycosylation for personalized medicine. Biochim Biophys Acta. 2016; 1860(8):1583-1595.

2. Miura $Y$, Endo $T$. Glycomics and glycoproteomics focused on aging and age-related diseases-Glycans as a potential biomarker for physiological alterations. Biochim Biophys Acta. 2016; 1860(8):1608-1614.

3. Baxendale S, van Eeden F, Wilkinson R. The Power of Zebrafish in Personalized Medicine. Adv Exp Med Biol. 2017; 1007:179-197.

4. Balfour-Lynn IM. Personalized medicine in cystic fibrosis is unaffordable. Paediatr Respir Rev. 2014; 15(1):2-5.

5. Savard J. Personalized medicine: a critique on the future of health care. J Bioeth Inq.2013; 10(2):197-203.

6. Brittain HK, Scott $R$, Thomas $E$. The rise of the genome and personalized medicine. Clinical Medicine. 2017; 17(6): 545-551.

7. Dalbeth N, Stamp LK, Merriman TR. The genetics of gout: towards personalized medicine? BMC Medicine. 2017; 15:108.

8. Hov JE, Trøseid M. Personalized medicine targeting the gut microbiota? Tidsskr Nor Laegeforen. 2015; 135(7):624.

9. Day S, Coombes RC, McGrath-Lone L, et al. Stratified, precision or personalized medicine? Cancer services in the 'real world' of a London hospital. Sociol Health IIIn. 2017; 39(1):143-158.

10. Arjmand B, Goodarzi P, Mohamadi-Jahani F. Personalized Regenerative Medicine. Acta Medica Iranica. 2017; 55(3):144-149.

11. Krasnewich D. Human glycosylation disorders. Cancer Biomarkers. 2014; 14:3-16.

12. Schnaar RL. Glycobiology simplified: diverse roles of glycan recognition in inflammation. Journal of Leukocyte Biology. 2016; 99(6):825-838.

13. Adkar SS, Brunger GM, Willard VP, et al. Genome Engineering for Personalized Arthritis Therapeutics. Trends Mol Med. 2017; 23(10): 917-931.

14. Kavanaugh $D, O^{\prime}$ Callaghan J, Kilcoyne M. The intestinal glycome and its modulation by diet and nutrition. Nutrition Reviews. 2015; 73(6):359-375.

15. Zenner HP. Individual Biomarkers Using Molecular Personalized Medicine Approaches. ORL J Otorhinolaryngol Relat Spec. 2017; 79(1-2):7-13.

16. Shukla SK, Murali NS, Brilliant MH. Personalized Medicine Going Precise: From Genomics to Microbiomics. Trends Mol Med. 2015; 21(8):461-462.

17. Hartmann J. Clinical development of CAR T cells - challenges and opportunities in translating innovative treatment concepts. EMBO Mol Med. 2017; 9(9):1183-1197.

18. Ren J, Zhao Y. Advancing chimeric antigen receptor T cell therapy with CRISPR/Cas9. Protein Cell. 2017; 8(9):634-643.

19. https://clinicaltrials.gov/

20. Jackson SE, Chester JD. Personalized cancer medicine. Int J Cancer. 2015; 137:262-266.

21. Marotz CA, Zarrinpar A. Treating Obesity and Metabolic Syndrome with Fecal Microbiota Transplantation. Yale J Biol Med. 2016; 89(3):383-388.

22. Gilbert B, Schrenzel J. Fecal microbiota transplantation: current status and prospect. Rev Med Suisse. 2019; 15(650):976-983.

23. Chang CS, Ruan JW, Kao CY. An overview of microbiome based strategies on anti-obesity. Kaohsiung J Med Sci. 2019; 35(1):7-16.

24. Tavassoly I, Hu $Y$, Zhao $S$, et al. Genomic signatures defining responsiveness to allopurinol and combination therapy for lung cancer identified by systems therapeutics analyses. Mol Oncol. 2019. DOI: 10.1002/1878-0261.12521. [Epub ahead of print].

25. El-Deiry WS, Goldberg RM, Lenz HJ, et al. The current state of molecular testing in the treatment of patients with solid tumors. CA Cancer J Clin. 2019. DOI: 10.3322/ caac.21560. [Epub ahead of print].

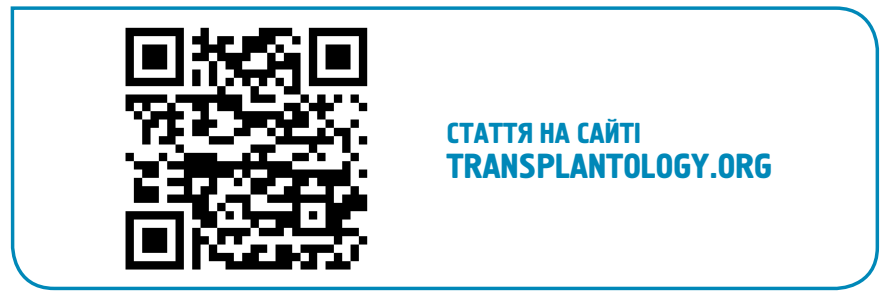

